\title{
Identificando as Preferências do Banco Central do Brasil (2002-2013)
}

\author{
Thiago Curado \\ Mestrando em Economia (EESP-FGV) \\ Endereço: Rua Itapeva, 474 - Bela Vista - São Paulo/SP - Brasil \\ CEP: 01332-000 - E-mail: tlcurado@gmail.com
}

\author{
Marcelo Curado \\ Professor - Universidade Federal do Paraná (UFPR) \\ Endereço: Endereço: Avenida Lothario Meissner, 632 - Jardim Botânico - Curitiba/PR - Brasil \\ CEP: 80210-070 - E-mail: mlcurado@gmail.com
}

Recebido em 19 de setembro de 2013. Aceito em 15 de abril de 2014.

\begin{abstract}
Resumo
Este trabalho estima os parâmetros de preferência do Banco Central do Brasil para o período compreendido entre 2002 e 2013. Para isso, analisamos um problema de otimização do Banco Central em uma economia aberta. A equação de Euler que soluciona tal problema permite utilizar o método generalizado dos momentos (GMM) para estimação eficiente dos parâmetros. Tal abordagem separa os parâmetros de preferência daqueles relacionados às condições estruturais da economia brasileira, ao contrário do que ocorre na estimação direta de uma regra de política monetária. Os resultados encontrados indicam grau relativamente elevado de flexibilidade do regime de metas de inflação no Brasil, sugerindo que a estabilização do produto em torno de seu potencial constitui objetivo relevante nas decisões de política monetária, em detrimento ao controle inflacionário estrito. A flexibilidade do regime parece ter sido aprofundada na gestão de Alexandre Tombini à frente do Banco Central do Brasil.
\end{abstract}

\section{Palavras-Chave}

regras de política monetária, metas de inflação, preferências do Banco Central

\begin{abstract}
This paper estimates Central Bank's preferences for the period between 2002 and 2013. Therefore, we analyze a Central Bank optimization problem within an open economy. The Euler equation that solves this problem allows to apply the generalized method of moments (GMM) to efficiently estimate the parameters. Such an approach dissociates the preference's parameters from those related to the structural conditions of Brazilian economy, unlike what occurs on direct estimations of a monetary policy rule. The results indicate a high degree of flexibility in Brazil's inflation targeting regime, suggesting that
\end{abstract}

- Agradecemos a Márcio Nakane, cuja orientação foi essencial nas etapas iniciais desse trabalho, e aos revisores da revista Estudo Econômicos por suas sugestões. Também somos gratos à equipe da "Tendências Consultoria Integrada", em especial Adriana Molinari, que nos auxiliou na obtenção e no processo de tratamento dos dados. As imprecisões remanescentes são de responsabilidade exclusiva dos autores. 
output stabilization around its potential is a significant goal in monetary policy decisions, over a strict inflation control. This flexible nature of the monetary policy management seems to have been deepened over the administration of President Alexandre Tombini.

\section{Keywords}

interest rate rules, inflation targeting, Central Bank Preferences.

\section{JEL Classification}

E52, C26, C51

\section{Introdução}

Svensson (1997), Bernanke et al. (1999) e Bogdanski et al. (2000) definem um regime metas de inflação como aquele no qual as ações da política monetária, sobretudo a fixação da taxa de juros básica, são guiadas com o objetivo explícito de obtenção de uma taxa de inflação (ou de nível de preços) previamente determinado. ${ }^{1}$

Há vasta literatura empírica que busca, através da estimação direta da regra de Taylor, analisar o comprometimento da política monetária em alcançar a meta de inflação. No campo internacional, Eichenbaum e Evans (1995), Cristiano et al. (1996), Clarida et al. (1998), Kuttner e Posen (1999), Nelson (2000) e Newmann e Von Hagen (2002), entre outros, são exemplos de trabalhos que estimam versões modificadas da regras de Taylor com intuito de analisar o grau de rigidez da política monetária. No campo doméstico, os trabalhos de Minella et al. (2002), Holland (2005), Mendonça (2007) e Modenesi (2011) são exemplos da literatura da área.

Minella et al. (2002) estimam funções de reação para o Banco Central do Brasil (BACEN) do tipo forward-looking, utilizando dados mensais de julho de 1999 a junho de 2002. Os autores concluem que, a partir da adoção do regime de metas de inflação, o BACEN teve comportamento forward-looking e respondeu de forma agressiva aos desvios entre a expectativa de inflação e a meta. Holland (2005) conclui, estimando funções de reação a partir da adoção do regime de metas no Brasil, que o BACEN tem uma postura agressiva de controle de inflação, fato constatado pelos valores dos coeficientes de inflação estimados (consideravelmente superiores

1 No Brasil, o regime foi formalmente adotado no dia primeiro de julho de 1999. Decreto presidencial no. 3088 e resolução $n^{\circ}$. 2615 do Conselho Monetário Nacional (CMN) 
à unidade e estatisticamente significantes). Mendonça (2007) apresenta os resultados estimados separando os efeitos das variações da taxa de juros sobre os preços livres e os administrados. Finalmente, Modenesi (2011) estima a regra de Taylor para o Brasil entre 2000 e 2007. A conclusão central do trabalho é que a política anti-inflacionária tem resultado em elevadíssima taxa de sacrifício, fruto da rigidez com que foi conduzida a política monetária no período.

No entanto, os coeficientes obtidos pela estimação direta de uma regra de política monetária, tal como o realizado pela literatura brasileira, podem ser interpretados apenas como uma sobreposição dos parâmetros que efetivamente descrevem as preferências do Banco Central e daqueles que caracterizam os aspectos estruturais da economia. Assim, a estimação direta de uma regra de Taylor comete o equívoco de confundir tais características, podendo levar a avaliações enganosas sobre os padrões de decisão de política monetária. Como argumentam Favero e Rovelli (2003) na abordagem tradicional da regra de Taylor "as regras de acordo com as quais os Bancos Centrais definem as taxas de juros são discutidas como se fossem independentes da estrutura da economia e dos mecanismos de transmissão da política monetária." ${ }^{2}$

A abordagem utilizada neste artigo diferencia-se desta literatura ao estimar as preferências do Banco Central a partir de um problema de otimização, o que nos permite separar explicitamente os parâmetros estruturais dos parâmetros de preferência. Consideramos essa diferenciação particularmente importante para o caso brasileiro, uma vez que o caráter relativamente recente da conquista de estabilidade macroeconômica faz com que os canais de transmissão da política monetária encontrem-se sob rápida e constante adaptação. Além disso, tal especificação permite incorporar o papel de flutuações da taxa de câmbio nas decisões de política monetária (fear of floating).

Para evitar o efeito de sobreposição de parâmetros, aplicamos a metodologia inicialmente proposta por Svensson (1997) e ampliada por Favero e Rovelli (2003) e D’Adamo (2010), ao invés de estimarmos diretamente uma regra de Taylor em sua forma tradicional.Svensson (1997) observou que a equação representativa da regra de política monetária poderia ser obtida solucionando um problema de otimização do Banco Central. Em linhas gerais, a ideia é que o Banco

\footnotetext{
2 Favero e Rovelli (2003), página 5.
} 
Central minimize uma função de perda, tendo como restrição de atuação a estrutura geral da economia e os mecanismos de transmissão da política monetária, explícitas formalmente por uma curva IS, uma curva de Phillips e uma equação de paridade descoberta de juros. Nesse arcabouço, a utilização dos métodos generalizados dos momentos (GMM) constitui caminho natural para estimação dos parâmetros de preferência do Banco Central. Isso porque a derivação da equação de Euler subjacente ao problema de otimização resulta na obtenção de uma condição de momento a partir da qual podemos empregar diretamente o GMM. ${ }^{3}$

Em síntese, o objetivo central deste artigo é estimar os parâmetros de preferência do Banco Central como um problema de otimização, de forma a identificar possíveis desvios do padrão de condução da taxa de juros daquele teoricamente esperado em um regime de metas de inflação "puro". Mais especificamente, procuramos testar a hipótese de alteração das preferências do Banco Central no passado recente. Além deste objetivo, pretende-se também discutir a hipótese de que o Banco Central é sensível a riscos de estouro do limite superior da banda, apesar de não perseguir a convergência da inflação ao centro da meta oficial com o vigor esperado no regime de metas de inflação. Colocando em outros termos, isso significaria que a meta de jure diverge da meta de facto. Para isso, avaliamos as implicações sobre os parâmetros de preferência do Banco Central na medida em que trabalhamos com metas alternativas à oficialmente estabelecida.

Para atingir os objetivos propostos, o artigo organiza-se da seguinte forma. Após essa introdução, a seção 2 apresenta o modelo teórico utilizado no trabalho. Na sequência, a seção 3 discute o método de estimação empregado, enquanto a seção 4 apresenta os principais resultados obtidos. A seção 5 conclui o trabalho.

3 Nesse ponto, seguimos a argumentação de D'Adamo (2010), pg.8. As a natural way to estimate the model, we implement GMM on the first order conditions choosing as instruments for expected inflation those variables which are consistent with the dynamic specification of equations. 


\section{Modelo para Economia Brasileira}

Essa seção baseia-se essencialmente nos trabalhos de Svensson (1997), Favero e Rovelli (2003) e D'Adamo (2010). Favero e Rovelli (2003) utilizaram um modelo de economia fechada para estudar o padrão de comportamento da política monetária dos Estados Unidos entre 1961 e 1998, com o objetivo de identificar mudanças de preferência do Federal Reserve na era Volcker-Greenspan. A contribuição de D'Adamo (2010) foi ampliar o modelo de Favero e Rovelli (2003) para o caso de uma pequena economia aberta.

A forma mais geral da função de perda $\mathcal{L}$ do Banco Central pode ser apresentada como: ${ }^{4}$

$$
\begin{aligned}
& \mathcal{L}=E_{t} \sum_{k=0}^{T} \delta^{k}\left[\lambda_{\pi}\left(\pi_{t+k}-\bar{\pi}_{t+k}\right)^{2}+\lambda_{y} y_{t+k}^{2}+\lambda_{i}\left(i_{t+k}-i_{t+k-1}\right)^{2}+\right. \\
& \left.\lambda_{r}\left(i_{t+k}-\pi_{t+k}-r\right)^{2}+\lambda_{e}\left(e_{t+k}-e_{t+k-1}\right)^{2}\right]
\end{aligned}
$$

Onde $\delta^{\kappa}$ é a taxa de desconto intertemporal, $\pi_{t}$ é a inflação realizada, $\bar{\pi}_{t}$ é a meta de inflação oficial, $y_{t}$ o hiato do produto, $i_{t}$ é a taxa de juros, $r$ é a taxa de juros real de equilíbrio e $e_{t}$ é a taxa de câmbio nominal. ${ }^{5}$ Pode-se argumentar que a função perda assim apresentada é, de certa forma, ad hoc. No entanto, como demonstrado por Rotemberg e Woodford (1998), uma função de tal forma pode ser obtida a partir uma aproximação quadrática de uma função de bem - estar baseada num problema clássico de maximização de utilidade intertemporal. ${ }^{6}$

A estrutura de preferências do Banco Central é determinada pelos parâmetros $\lambda$. Assim, temos que $\lambda_{\pi}$ é o peso atribuído aos desvios quadráticos da inflação em relação à meta oficial, enquanto $\lambda_{y}$ é o peso dos desvios do produto em relação ao seu nível potencial. Já o parâmetro $\lambda_{i}$ representa o peso atribuído à suavização da taxa de juros, incorporando ao modelo o caráter tipicamente gradual dos processos de ajustes das condições monetárias. De forma similar, $\lambda_{e}$ é o peso atribuído à suavização da taxa de câmbio nominal. A inclusão do termo $\lambda_{e}$ é justificada pela literatura de Fear of Floating, fenômeno inicialmente descrito por Calvo e Reinhard (2002).

4 D'Adamo (2010).

5 A descrição detalhada de cada uma dessas variáveis encontra-se em anexo.

${ }^{6}$ Mais precisamente, Rotemberg e Woodford (1998) utilizam um modelo em que um contínuo de agentes interagem sob uma estrutura competição monopolística com rigidez de preço a la Calvo. 
Em nossa especificação, optamos por desconsiderar o parâmetro $\lambda_{r}$, que representa o peso dado à estabilização da taxa de juros real ao redor do patamar de equilíbrio. Para o caso da economia brasileira, tal abordagem é particularmente dificultada pela trajetória cadente do juros real de equilíbrio ao longo do período estudado, o que não nos permitiria assumir como constante tal nível de equilíbrio. A alternativa a essa hipótese seria estimar a trajetória trimestral do taxa de juros real de equilíbrio. Todavia, tal caminho exigiria um estudo específico mais aprofundado sobre o comportamento da variável, o que foge ao escopo desse trabalho. Ademais, correríamos o risco de adicionar ruídos a pontos mais importantes para nosso estudo, como a questão da evolução do parâmetro de aversão a desvios do produto.

A Tabela 1, adaptada de D'Adamo (2010), resume as diferentes possibilidades de regimes monetários. Em um regime de meta de taxa de câmbio esperaríamos observar peso um para $\lambda_{e}$ e zero para os demais parâmetros. Em outros termos, teríamos um sistema em que o Banco Central persiga um câmbio fixo, independentemente da evolução das demais variáveis macroeconômicas. Para os demais tipos de regime analisados, faremos a imposição de que $\lambda_{\pi}=1$. Isso permite comparar diretamente o peso atribuído pelo Banco Central aos demais objetivos de política monetária com o objetivo de estabilização da inflação em torno da meta. Assim, em um regime de meta de inflação estrita, o peso de todos os parâmetros que não $\lambda_{\pi}$ seriam iguais a zero, ou seja, as decisões de política monetária responderiam exclusivamente a desvios da taxa de inflação.

Tabela 1 - Parâmetros de preferência e regimes de política monetária

\begin{tabular}{|l|c|c|c|c|}
\hline \multicolumn{1}{|c|}{ Regime } & $\lambda_{\pi}$ & $\lambda_{y}$ & $\lambda_{i}$ & $\lambda_{e}$ \\
\hline Meta de taxa de câmbio & 0 & 0 & 0 & 1 \\
\hline Meta de inflação estrita & 1 & 0 & 0 & 0 \\
\hline Meta de inflação com suavização de juros & 1 & 0 & $>0$ & 0 \\
\hline Meta de inflação flexível & 1 & $\geq 0$ & $\geq 0$ & 0 \\
\hline Caso Geral (Fear of Floating) & 1 & $\geq 0$ & $\geq 0$ & $>0$ \\
\hline
\end{tabular}

Fonte: D'Adamo (2010)

O terceiro caso, meta de inflação com suavização de juros, aproxima-se bastante do anterior: a inflação segue sendo a única variável relevante para a política monetária. Todavia, os ajustes nas taxas de 
juros realizados para lidar com desvios da inflação passam a ser feitos de forma gradual, dada a existência de preferência pela suavização da taxa de juros $\left(\lambda_{i}>0\right)$.

Já no que chamamos de meta de inflação flexível, questões relacionadas à atividade econômica começam a ganhar relevância nas decisões de política monetária. Em situações em que o produto encontre-se abaixo de seu potencial, o Banco Central pode ter incentivo a reduzir a taxa de juros, na tentativa de aquecer a atividade econômica por meio do relaxamento das condições de crédito. Se a prioridade do Banco Central, nesse caso, continua a ser a questão inflacionária dependerá se $\lambda_{y}$ é maior ou menor do que um.

Por fim, o regime aqui tratado como Caso Geral adiciona o componente de fear of floating ao caso anterior. Os modelos estimados na seção 5 partem desse Caso Geral; na sequência, os resultados empiricamente obtidos para os parâmetros de preferências são utilizados para avaliação do regime de política monetária de facto encontrado para o Brasil.

\section{Método de Estimação}

A obtenção dos parâmetros de preferência é feita a partir minimização da função de perda do Banco Central, sob restrição das estruturas de oferta e demanda da economia, representadas aqui por uma curva de IS e uma curva de Phillips. Assim, o problema do Banco Central pode ser descrito como:

$\min _{i_{t}} \mathcal{L}$ sujeito a:

$$
\begin{gathered}
y_{t}=d_{0}+d_{1}(L) y_{t-1}-d_{2}(L)\left(i_{t-1}-\pi_{t-1}\right)+d_{3}(L) q_{t-1}+d_{4}(L) \varpi_{t}+\epsilon_{t}^{y} \\
\pi_{t}=f_{0}+f_{1}(L) \pi_{t-1}+f_{2}(L) y_{t-1}+f_{3}(L) \Delta_{4} e_{t} C_{t}+\epsilon_{t}^{\pi} \\
q_{t}=e_{t}\left(p_{t}^{f} / p_{t}\right) \\
e_{t}=i_{t}^{f}-i_{t}+e_{t+1 \mid t}+\epsilon_{t}^{e}
\end{gathered}
$$


Onde: $\mathrm{q}_{\mathrm{t}}$ é a taxa de câmbio real, $\varpi_{\mathrm{t}}$ é a taxa de crescimento mundial, $\mathrm{C}_{\mathrm{t}}$ é um índice de preços externos, cotado em dólares, $i_{t}^{f}$ é a taxa de juros externa, $p_{t}^{f}$ é o nível de preços externos e $p_{t}$ é o nível de preços domésticos. $\epsilon_{t}^{y}, \epsilon_{t}^{\pi}$ e $\epsilon_{t}^{\mathrm{q}}$ são ruídos brancos. As demais variáveis seguem as definições anteriores. As estruturas de defasagens para cada variável explicativa foram obtidas empiricamente, conforme procedimento explicitado na próxima seção.

Essa estrutura de equações descreve os canais de influência da política monetária sobre a atividade real e sobre a inflação. Primeiramente, a curva IS (3.1) incorpora os impactos da taxa de juros real sobre o hiato do produto. Por sua vez, a curva de Phillips (3.2) avalia a transmissão de pressões do hiato do produto sobre a taxa de inflação. A política monetária afeta a inflação ainda por meio dos preços de produtos importados, dado seus impactos sobre a taxa de câmbio nominal definido pela Equação (3.4). Por fim, a Equação (3.3) descreve a dinâmica da taxa de câmbio real, que no curto prazo pode ser afetada por flutuações na taxa de juros nominal.

Para obtermos a contrapartida empírica das equações estruturais, estimamos separadamente as Equações (3.1) e (3.2), utilizando o Método de Mínimos Quadrados Ordinários (MQO). Destacamos que todas as variáveis empregadas ou não possuem padrão sazonal ou foram dessazonalizadas. Tais procedimentos estão descritos na seção Al do Anexo.

Além disso, destacamos que todas as variáveis empregadas são estacionárias. ${ }^{7}$ Para garantirmos isso, empregamos o teste de raiz unitária de Phillips e Perron em cada uma das variáveis (nas formas funcionais utilizadas nas estimações). As variáveis $\pi_{t+\mathrm{k}}$ e $y_{\mathrm{t}+\mathrm{k}}$ rejeitaram a $5 \%$ a hipótese nula de existência de raiz unitária. Todas as demais variáveis rejeitaram a $1 \%$ a hipótese nula. Os valores encontrados para a estatística de Phillips-Perron e os p-valores do teste de hipótese encontram-se discriminados na Tabela 2.

7 Inicialmente, tivemos aqui um problema de não estacionariedade da série de juros real, dada sua trajetória cadente no período estudado. Para contornarmos tal problema, a variável incluída na estimação da curva IS foi a primeira diferença da taxa real, conforme explicitado pela Equação (3.1). Como em nossa IS incluímos como variável explicativa o hiato do produto defasado com coeficiente igual à um, tal formulação implica que estamos explicando variações do hiato do produto a partir de variações do patamar da taxa de juros real. 
Todas as defasagens não significativas a 5\% foram excluídas do modelo. Os critérios tradicionais de seleção ótima de defasagens foram utilizados para se chegar à especificação final das equações. ${ }^{8}$ Os valores exatos estimados para os parâmetros e os erros-padrão estimados encontram-se discriminados na Tabela 2.

$$
\begin{aligned}
& y_{t+k}=y_{t+k-1}+\beta_{1}\left(r_{t+k-2}-r_{t+k-3}\right)+ \\
& \beta_{2}\left(q_{t+k-2}\right)+\beta_{3}\left(w_{t+k}-w_{t+k-1}\right)+\epsilon_{t+k}^{y} \\
& \pi_{t+k}=\pi_{t+k-1}+\alpha_{1}\left(y_{t+k-2}\right)+ \\
& \alpha_{2} e_{t+k-2} C_{t+k-2}+\epsilon_{t+k}^{\pi}
\end{aligned}
$$

\begin{tabular}{|c|c|c|c|c|c|}
\hline & \multicolumn{3}{|c|}{ Equações Estruturais } & \multicolumn{2}{|c|}{ Teste de Phillips-Perron } \\
\hline & Variável & $\begin{array}{l}\text { Coeficiente } \\
\text { Modelo }\end{array}$ & $\begin{array}{c}\text { Coeficiente } \\
\text { Estimado }\end{array}$ & Estatística Observada & P-Valor \\
\hline \multirow{4}{*}{ (3.1') Curva IS } & $y_{t+k}-y_{t+k-1}$ & & & -4.6554 & $0.05 \%$ \\
\hline & $r_{t+k-2}-r_{t+k-3}$ & $\beta_{1}$ & $\begin{array}{c}-0.3799^{* *} \\
(0,1590)\end{array}$ & -4.9337 & 0.02 \\
\hline & $q_{t+k-2}$ & $\beta_{2}$ & $\begin{array}{l}-0.0507^{*} \\
(0.0156)\end{array}$ & -5.7665 & 0.00 \\
\hline & $w_{t+k}-w_{t+k-1}$ & $\beta_{3}$ & $\begin{array}{l}0.3886^{*} \\
(0.1289)\end{array}$ & -3.7604 & $0.64 \%$ \\
\hline \multirow{2}{*}{$\begin{array}{l}\text { (3.2') Curva de } \\
\text { Phillips }\end{array}$} & $\begin{array}{l}\pi_{t+k}-\pi_{t+k-1} \\
y_{t+k-2}\end{array}$ & $\alpha_{1}$ & $\begin{array}{l}0.3075^{\star} \\
(0.1135)\end{array}$ & $\begin{array}{l}-2.9467 \\
-3.06 a 24\end{array}$ & $\begin{array}{l}.83 \% \\
3.68 \%\end{array}$ \\
\hline & $e_{t+k-2} C_{t+k-2}$ & $\alpha_{2}$ & $\begin{array}{l}0.0424^{* *} \\
(0.0208)\end{array}$ & -3.9799 & 0.34 \\
\hline
\end{tabular}

Tabela 2 - Resultados da estimação e Testes de raiz unitária

Amostra: 44 observações, compreendidas entre o $2^{\circ}$ Trimestre de 2003 e $2^{\circ}$ trimestre. * parâmetros significativos à 1.0\%. ** parâmetros significativos à $5.0 \%$.

Erros padrão entre parênteses.

Valores críticos teóricos da estatística teste de Phillips-Perron: 1\%: -3,5925. 5\%: -2,9314. 10\%:-2.6039

Para estimarmos os parâmetros de preferência do Banco Central, primeiramente minimizamos a função perda $\mathcal{L}$ sujeita às restrições (3.3), (3.4), (3.1') e (3.2'). A equação de Euler que soluciona tal problema constitui o momento utilizado em nossa estimação dos parâmetros de preferência. Aqui, é útil nos atermos um pouco mais

8 Para seleção das defasagens empregadas, avaliamos o Critério de Informação de Akaike (AIC) e o diagnóstico dos resíduos do modelo (FAC, FACP, Ljung-Box e teste de normalidade). 
sobre tal metodologia. De acordo com Parker (2007) "uma equação de Euler é uma equação diferencial ou em diferenças que constitui a condição intertemporal para um problema dinâmico de escolha, descrevendo a evolução das variáveis ao longo da trajetória ótima." 9

Em nosso caso, a equação de Euler descreverá a trajetória ótima da taxa de juros, dada a dinâmica das variáveis macroeconômicas e os parâmetros de preferência do Banco Central. Além disso, "em um modelo com incerteza, a equação de Euler na forma de expectativas nos proverá diretamente condições de momento que podem ser usadas (...) para estimar os parâmetros do modelo, de forma que suas implicações quantitativas se aproximem ao máximo dos dados observados." (Parker, 2007, p.2)

Assim, a utilização dos métodos generalizados dos momentos (GMM) constitui caminho natural para estimação dos parâmetros de preferência do Banco Central, após a derivação da equação de Euler. Utilizamos uma abordagem de horizonte finito para a função de perda do Banco Central. Tal opção não nos parece ser excessivamente simplificadora, e nos permite a obtenção analítica das condições de primeira ordem. Além disso, assumimos que $\tau=4$, ou seja, assumimos que o Banco Central reage às expectativas de desvios da inflação e do produto um ano à frente. Este horizonte de tempo é suficiente para que os diversos canais de influência da política monetária sobre a inflação sejam concretizados. Assim, a ideia implícita em nossa abordagem é que, a cada trimestre, o Banco Central avalie a condição esperada para quatro trimestres à frente, e atue com base em tais previsões. Supor um $\tau<4$ foi descartado pela impossibilidade de interferência concreta das decisões de política monetária (i.e da taxa de juros) sobre as condições macroeconômicas.

Também precisamos definir a taxa de desconto intertemporal, $\delta$. Para esse fim, definimos $\delta=0,984$, em linha com o a definição de D'Adamo (2010). Favero e Rovelli (2003) também impõem um valor específico para a taxa de desconto, fixando $\delta=0,975$. Os resultados apresentados na seção 5 mostraram-se robustos para este último valor. Resolvendo o problema do Banco Central, chegamos à seguinte condição de momento, que será utilizada em nossa estimação dos parâmetros de preferência:

9 Parker (2007), página 1. 
$i_{t}-i_{t-1}+0.2268 \phi_{1}\left(\pi_{\mathrm{t}+4 \mid \mathrm{t}}-\pi\right)-$

$0.5998 \phi_{2} y_{t+4 \mid t} \phi_{2}-\phi_{3}\left(e_{t}-e_{t-1}\right)=0$

Onde $\phi_{1}=\frac{1}{\lambda_{i}}, \phi_{2}=\frac{\lambda_{y}}{\lambda_{i}}$ e $\phi_{3}=\frac{\lambda_{e}}{\lambda_{i}}$ Vale observar que utilizando tal formulação não encontramos nenhum problema para identificação dos parâmetros de preferência do Banco Central.

Os candidatos imediatos a instrumentos para as expectativas de inflação e hiato do produto são as variáveis consistentes com o modelo estrutural empírico, conforme argumentam Favero e Rovelli (2003). D’Adamo (2010) utiliza o mesmo critério para seleção de instrumentos. Mais especificamente, utilizamos como instrumentos na estimação do GMM: de uma a quatro defasagens do IPCA, crescimento mundial e hiato do produto, e de duas a quatro defasagens do câmbio real e do índice Commodity Research Bureau (CRB), excluindo o petróleo).

Uma última questão necessita ser apontada nesse momento. Como podemos observar, a Equação 3.4 tem a formulação típica de uma regra de Taylor tradicional. Todavia, os parâmetros presentes na regra de Taylor são resultantes da interação dos parâmetros de preferência do Banco Central com os parâmetros das equações estruturais da economia. Por exemplo, o parâmetro do termo dos desvios do produto é a multiplicação dos parâmetros de preferência $\frac{\lambda_{y}}{\lambda_{i}}$ por $k_{2}=\beta_{1}{ }^{2} \beta_{3}+\beta_{1}{ }^{2} \beta_{2}$, além da taxa de desconto intertemporal $\delta^{4}$. Assim, como notam Favero e Rovelli (2003) na conclusão de seu trabalho.

Uma vez que os parâmetros presentes na regra de política monetária são convoluções dos parâmetros "profundos" de preferência com aqueles que descrevem a estrutura da economia, não é possivel identificar tal regra a partir da estimação direta da equação de política monetária. De toda forma, as preferências são naturalmente identificadas pelas condições de primeira ordem do problema de otimização intertemporal defrontado pelo Banco Central, para uma dada estrutura da economia. (Favero e Rovelli, 2003, p. 10) 
Em síntese, utilizando a presente metodologia, conseguimos identificar diretamente os parâmetros $\lambda$, separadamente dos parâmetros derivados das equações estruturais. De um ponto de vista mais objetivo, o ganho obtido dessa forma é a possibilidade de diferenciação de mudanças de preferências e mudanças das condições macroeconômicas de um país. A estimação direta de uma regra de Taylor comete o equívoco de confundir tais efeitos, podendo levar a avaliações enganosas sobre as preferências dos Bancos Centrais. Consideramos essa diferenciação particularmente importante para o caso brasileiro, uma vez que o caráter relativamente recente da conquista de estabilidade macroeconômica faz com que os canais de transmissão da política monetária encontrem-se sob constante adaptação.

\section{Resultados Empíricos}

A motivação inicial de nosso trabalho foi testar a hipótese de que as decisões de política monetária estariam sendo cada vez mais pautadas por questões relativas à atividade econômica, em detrimento do controle inflacionário estrito que seria esperado em um regime de metas de inflação "puro". Em termos do nosso modelo, a hipótese é a de que o parâmetro $\lambda_{y}$, que representa o peso atribuído aos desvios do produto, relativamente ao peso atribuído à inflação (normalizado para um), estaria ganhando força nos últimos anos. De fato, os resultados empíricos apontam no sentido de confirmação de tal hipótese. A segunda coluna Tabela 3 apresenta os resultados para a estimação do modelo utilizando toda a amostra (do segundo trimestre de 2002 ao segundo trimestre de 2013).

Para garantirmos que todos os instrumentos utilizados de fato satisfazem as condições de ortogonalidades assumidas, utilizamos o teste de sobreidentificação de Hansen (estatística J). Em todas as estimações reportadas neste trabalho, a estatística J encontrada ficou distante da área de rejeição da hipótese nula, indicando que o conjunto de instrumentos utilizado é de fato apropriado. ${ }^{10} \mathrm{O}$ valor

10 A hipótese nula do teste é a de que os instrumentos são válidos (i.e., satisfazem a condição de ortogonalidade). Assim, se a estatística for significante $(p<0.10)$, temos um indicativo de que pelo menos um dos instrumentos não é válido. 
exato encontrado para as estatísticas J encontram-se na última linha da Tabela 3.

Os resultados indicam que o peso atribuído à suavização da taxa de juros é cerca de dois terços do peso atribuído à inflação, enquanto o parâmetro de suavização do câmbio nominal é cerca de treze vezes inferior à $\lambda_{\pi}$. Todavia, a estabilização do produto tem peso superior à estabilização da inflação. O resultado surpreende, uma vez que se desvia de maneira crítica do esperado para um país com regime formal de metas de inflação.

O resultado obtido diverge daqueles tipicamente encontrados nos trabalhos empíricos sobre a política monetária brasileira após a adoção do regime de metas, tal como, por exemplo, Modenesi (2011). As evidências empíricas encontradas permitem sustentar que o elevado patamar da taxa de juros brasileira, frente aos padrões internacionais, não deve ser justificado como resultado de um Banco Central excessivamente rígido no controle inflacionário, mas sim como fruto de condições estruturais de funcionamento da economia brasileira.

Uma hipótese importante a ser testada é de que, embora a manutenção da inflação em níveis próximos ao centro da meta de inflação não seja sempre priorizada nas decisões de política monetária, o Banco Central é sensível ao limite superior da banda. Para testar essa proposição, criamos duas variáveis alternativas para a meta de inflação. À primeira somamos 0.5 p.p. em todas as observações; nos referiremos a esse caso como meta ajustada 0.5. No segundo caso, somamos 1.0 p.p.; analogamente chamada de meta ajustada 1.0. Assim por exemplo, a estimação do modelo no primeiro caso considerará a meta como sendo de $5,0 \%$ para o período em que a meta oficial foi fixada em $4,5 \%$, enquanto no segundo caso a meta considerada será de $5.5 \%$. As demais configurações da estimação foram mantidas inalteradas. Os resultados de tal experimento nos parecem particularmente interessantes, e estão explicitados na segunda e terceira colunas da Tabela 3. 
Tabela 3: Resultados da estimação dos parâmetros de preferência

\begin{tabular}{|c|c|c|c|c|}
\hline & & Meta Oficial & Meta ajustada 0.5 & Meta ajustada 1.0 \\
\hline \multirow{2}{*}{ Parâmetros estruturais } & $k_{1}$ & -0.2268 & & \\
\hline & $k_{2}$ & -0.5998 & & \\
\hline \multirow{5}{*}{ Parâmetros diretamente obtidos na estimação } & $\phi_{1}$ & $\begin{array}{c}1.493^{*} \\
(0.377)\end{array}$ & $\begin{array}{l}2.994^{*} \\
(0.190)\end{array}$ & $\begin{array}{l}3.351^{\star} \\
(0.126)\end{array}$ \\
\hline & & $2.000^{*}$ & $1.864^{*}$ & $0.325^{\star *}$ \\
\hline & $\phi_{2}$ & $(0.389)$ & $(0.271)$ & $(0.136)$ \\
\hline & $\phi_{3}$ & $0.111^{\star}$ & $0.167^{*}$ & $0.087^{*}$ \\
\hline & & $(0.021)$ & $(0.025)$ & $(0.009)$ \\
\hline \multirow{4}{*}{ Parâmetros de Preferência Implícitos } & $\lambda_{\pi}$ & 1.000 & 1.000 & 1.000 \\
\hline & $\lambda_{\mathrm{i}}$ & 0.670 & 0.334 & 0.298 \\
\hline & $\lambda_{y}$ & 1.340 & 0.623 & 0.097 \\
\hline & $\lambda_{\mathrm{e}}$ & 0.074 & 0.056 & 0.026 \\
\hline Estatística J de Hansen - Chi-Sq (16) & & 8.817 & 4.296 & 4.044 \\
\hline
\end{tabular}

Instrumentos utilizados: $\pi_{t-1}, \ldots, \pi_{t-4} ; y_{t-1}, \ldots, y_{t-4} ; \varpi_{t-1}, \ldots, \varpi_{t-4} ; C_{t-2}, \ldots, C_{t-4} ; q_{t-2}, \ldots, q_{t-4}$.

Erros padrão HAC entre parênteses.

* parâmetros significativos à $1.0 \%{ }^{* *}$ parâmetros significativos à 5.0\%.

O emprego da variável meta_ajustada_0.5 já implica alterações relevantes nos parâmetros estimados. O parâmetro $\lambda_{\mathrm{y}}$ tem seu peso reduzido para 0.623 , isto é, o peso dado a desvios do produto perde a predominância frente ao controle inflacionário. A relevância do fear of floating continua a ser reduzida (0.056), ao passo que $\lambda_{i}$ perde metade sua importância relativa. Ou seja, ao deslocarmos para cima em 0.5 p.p. a variável da meta de inflação, nosso modelo passa a identificar um regime de metas de inflação menos flexível.

A alteração se torna ainda mais significativa para o caso da meta ajustada_1.0. O peso atribuído aos desvios do produto passa a representar apenas $10 \%$ do peso atribuído a desvios da inflação. Ao mesmo tempo, $\lambda_{i}$ e $\lambda_{e}$ seguem perdendo importância relativa, sendo estimados em 0.298 e 0.026 , respectivamente. Em suma, se considerarmos a meta de inflação de facto como sendo sempre 1.0 p.p. acima da meta de jure (i.e, com a meta passando a ser a mediana entre o target oficial e o limite superior da banda) nossa estimação passa a identificar um padrão de gestão de política monetária que se aproxima do esperado em regimes de metas de inflação "puros".

Esse último resultado (meta_ajustada_1.0) é o que mais se aproxima, no tocante ao parâmetro $\lambda_{y}$, daqueles obtidos pela literatura internacional. D'Adamo (2010) encontra que o parâmetro de aversão 
a desvios do produto, $\lambda_{y}$ é não significativo nos níveis tradicionais. Já o coeficiente de suavização de juros $\lambda_{i}$ encontrado foi maior do que o nosso, ficando em 0.493. Por outro lado, o parâmetro de fear of floating encontrado para o caso brasileiro é idêntico ao observado para a Suécia, sendo significativo, mas representando apenas $2.6 \%$ do peso atribuído a desvios da inflação.

Já Favero e Rovelli (2003) estimam seu modelo para duas sub amostras: primeiro trimestre de 1961 ao terceiro trimestre de 1979, e terceiro trimestre de 1980 ao terceiro trimestre de 1998. Os autores encontram que os pesos de suavização da taxa de juros e de estabilização do produto são pequenos, mas significativos em ambos os casos. O parâmetro $\lambda_{i}$ é estimado em 0.0051 para a primeira sub amostra e em 0.0085 para a segunda. Já $\lambda_{y}$ é reduzido de 0.00153 para 0.00125 .

Falta ainda respondermos uma última pergunta: há evidências de que o regime de metas terá sido flexibilizado ao longo da gestão Tombini (que assumiu a presidência do Banco Central no primeiro trimestre de 2011)? Para responder a indagação reestimamos os parâmetros de preferência dez vezes, excluindo sucessivamente as últimas observações. Dessa forma, fomos reduzindo o peso dado na estimação total ao comportamento da atual gestão. A Figura 1 apresenta o resultado de tal procedimento. O eixo horizontal indica qual foi a última observação incluída em nossa estimação.

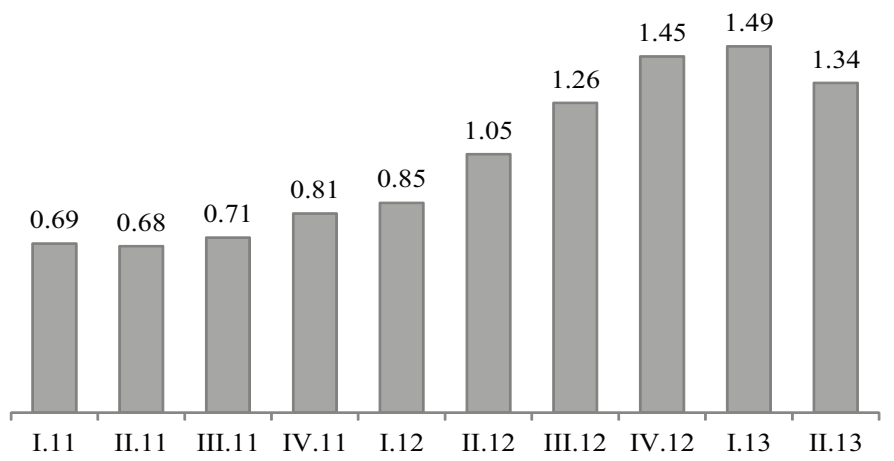

Figura 1: Evolução do parâmetro de aversão a desvios do produto 
Fica evidente a progressiva importância dada aos desvios do produto, relativamente a desvios da inflação, na medida em que incluímos em nossa amostra as decisões tomadas sob a presidência de Tombini, especialmente entre o último trimestre de 2011 e o primeiro trimestre de 2013. De fato, durante esse período ocorreram sucessivas reduções da taxa básica de juros com o intuito de incentivar a atividade econômica, que mostrava sinais nítidos de enfraquecimento, a despeito de expectativas de inflação elevadas, acima do centro da meta oficial. Por conta de tal padrão, nosso modelo capta contínuos incrementos de $\lambda_{y}$.

É interessante notar, no entanto, que o parâmetro de aversão a desvios do produto muda de trajetória no segundo trimestre de 2013. De fato, nesse período o Banco Central voltou a elevar a taxa Selic, a despeito de perspectivas ainda bastante desfavoráveis para a atividade econômica, em função da persistência de uma inflação em patamares muito elevados e de riscos reais de estouro da meta de inflação nos anos subsequentes.

\section{Considerações Finais}

Para avaliarmos o padrão de comportamento da condução de política monetária no passado recente, empregamos a metodologia inicialmente proposta por Svensson (1997) e ampliada por Favero e Rovelli (2003) e D'Adamo (2010). Dessa forma, conseguimos separar os parâmetros de preferência do Banco Central daqueles relacionados à estrutura da economia, elemento que se constitui num avanço em relação à metodologia comumente empregada de estimação direta de uma regra de Taylor, tal como realizado, entre outros, por Minella et al. (2002), Holland (2005), Mendonça (2007) e Modenesi (2011).

Os resultados obtidos apontam para um regime de metas de inflação mais flexível no Brasil do que o observado por Favero e Rovelli (2003) para os Estados Unidos e por D’Adamo (2010) para a Suécia. Nesse sentido, a estabilização do produto em torno de seu potencial parece constituir um objetivo importante nas decisões de política monetária tomadas pelo Banco Central do Brasil, em detrimento do controle inflacionário estrito. 
Nossos resultados apontam ainda que esse caráter mais flexível do regime de metas de inflação foi aprofundado ao longo da gestão de Alexandre Tombini.

Um segundo exercício apontou ainda para a possibilidade da meta de inflação de facto divergir da de jure. Assim, se considerarmos que o nível de inflação efetivamente perseguido pelo Banco Central é superior ao centro da meta oficial, observamos que o padrão de comportamento das decisões de política monetária se aproxima daquele esperado em um regime de metas de inflação estrito. Particularmente, se supusermos que a meta efetivamente perseguida é um ponto percentual acima da oficial, nosso modelo passa a diagnosticar um regime de inflação bastante rígido.

\section{Referências}

BERNANKE, B.; LAUBACH, T.; MISHKIN, F.;POSEN, A. Inflation Targeting. New Jersey: Princeton University Press, 1999.

BOGDANSKI, J.; TOMBINI, A. A.; WERLANG, S. R. Implementing inflation target in Brazil. Working Paper. Banco Central do Brasil, $n^{\circ} 1,2000$.

CALVO, G.A; REINHART, C.M. (2002). Fear of Floating. The Quarterly Journal of Economics, 118, Issue 2, p.379-407, 2002.

CHRISTIANO, L.; EICHENBAUM, M.; EVANS, C. Monetary Policy Shocks: What have we Learned and to what End? In: J. Taylor; M. Woodford, Handbook of Macroeconomics. North Holland: Elsevier Science, 1999.

CLARIDA, R, GALI, J.; GERTLER, M. Monetary Policy Rules in Practice: Some International Evidence. European Economic Review, 42, p. 1033-1067, 1998.

D’ADAMO, G. Estimating Central Bank Preferences in a Small Open Economy: Sweden 1995 - 2008. MPRA Paper No. 26575, 2010.

EICHENBAUM, M.; EVANS, C. Some Empirical Evidence on the Effects of Shocks to Monetary Policy on Exchange Rates. Quarterly Journal of Economics, 110 (4), p. 975-1009, 1995.

FAVERO, C.A; ROVELLI, R.. Macroeconomic Stability and the preferences of the FED. A formal analysis, 1961-9. Journal of Money, Credit and Banking 35, p. 545 - 556, 2003.

HOLLAND, M. Monetary and exchange rate policy after inflation. University of California, Berkeley, 2005.

KUTTNER, K; POSEN, A. Does Talk Matter After All? Inflation Targeting and Central Bank Behavior. Federal Reserve Bank of New York, Staff Reports 88, 1999.

MENDONÇA, H.F. Metas de inflação e taxa de juros no Brasil: uma análise do efeito dos preços livres e administrados. Revista de Economia Politica, 27, pp. 431-451, 2007.

MINELLA, A., GOLDFAJN, I.; MUINHOS, M. Inflation Targeting in Brazil: Lessons and Challenges. BIS Papers, 2002. 
MODENESI, A.D. Conservadorismo e rigidez na política monetária: uma estimativa da função de reação do BCB (2000-2007). Revista de Economia Politica, vol. 31, nº 3 (123), pp. 415-434, julho-setembro, 2011.

NEWMANN, M.J.M.; VON HAGEN, J. Does Inflation Targeting Matter? The Federal Reserve Bank of St Louis, 2002.

PARKER, J.A. Euler equations. New Palgrave Dictionary of Economics, 2007.

ROTEMBERG, J.J., WOODFORD, M. Interest rate rules in an estimated sticky price model. Monetary policy rules. University of Chicago Press, p. 57-126, 1999.

SILVA FILHO, T.N.C. Estimando o Produto Potencial Brasileiro: Uma Abordagem de Função de Produção. Working Paper. Banco Central do Brasil, 2001.

SVENSSON, L.E.O. Inflation forecast targeting: Implementing and monitoring inflation targets. European Economic Review, 41, p. 111-1146, 1997.

Open-economy inflation Targeting. Journal of International Economics 50, pg. 155-183, 2000

TAYLOR J.B. Discretion versus policy rules in practice. Carnegie-Rochester Conference Series on Public Policy, 39, pg. 195-214, 1993.

\section{Anexo 1 - Base de Dados}

Hiato do produto: $y_{t}$. Primeiramente, utilizamos a série oficial disponibilizada pelo IBGE da série encadeada do índice de volume trimestral do PIB, com ajuste sazonal. Em seguida, aplicamos o filtro HP sobre tal série. A medida utilizada como proxy para o hiato do produto foi calculada como a diferença percentual entre o número índice da série original e a série obtida após a aplicação do filtro HP.

Para garantirmos que tal variável constitui uma proxy adequada para o hiato do produto, fizemos a estimação do hiato também via função de produção. O ponto de partida para isso foi o trabalho para discussão de Silva Filho (2001). A especificação da função de produção testada foi uma Cobb-Douglas, com participação 0.5 do capital na renda. Os resultados mostraram poucas diferenças significativas entre o hiato do produto obtido por este último método e pela simples aplicação do filtro HP. Dessa forma, na versão final da estimação das equações estruturais utilizamos a série obtida pelo filtro HP, para manter plena consistência com a construção da variável de hiato esperado.

Para construção de $y_{t+4 \mid t}$, isto é, a medida de hiato esperado, inicialmente coletamos os dados, disponibilizados pelo Banco Central, referentes às expectativas de crescimento real anualizadas, trimestre a trimestre. A partir disso, construímos, também trimestre a trimes- 
tre, a expectativa para o PIB em número índice, aplicando sobre o PIB observado em $t$ a expectativa para o crescimento em $t+4$. $\mathrm{Na}$ sequência, essa primeira série obtida foi dessazonalizada, seguindo a metodologia oficial do IBGE para o cálculo das séries trimestrais do Sistema de Contas Nacionais descriminada pelo IBGE. Também seguindo o IBGE, o software empregado para o ajuste sazonal foi o X12-ARIMA, do Bureau de Census do Departamento de Comércio dos Estados Unidos. Tomamos ainda o cuidado de incluir o calendário de feriados brasileiros. Na sequência, aplicamos o filtro HP sobre os dados da série apenas até $t+4$, obtendo nossa estimativa de produto potencial. $\mathrm{O}$ procedimento foi repetido sucessivas vezes, incluindo um trimestre por vez. A justificativa para tal procedimento foi replicar da forma mais verossímil possível o hiato do produto que seria considerado pela autoridade monetária a cada momento do tempo. Por isso não aplicamos diretamente o filtro HP sobre a série completa. Nossos exercícios apontaram que tal cuidado traz impactos relevantes sobre os resultados finais da estimação.

Taxa de juros nominal: $i_{t}$. Primeiramente, coletamos os dados diários estimulados como meta definida pelo para a taxa Selic, em termos de percentual anual. A partir desses dados diários, calculamos a média trimestral, que foi empregada em nossas estimativas.

Taxa de câmbio real: $q_{t}$. Utilizamos o índice da taxa de câmbio efetiva real, deflacionada pelo IPA-DI, calculada pelo DEPEC-BACEN.

Inflação: $\pi_{t}$. Taxa de variação acumulada em doze meses pelo IPCA (Índice de Preços ao Consumidor Amplo), calculada pelo IBGE e utilizada como medida oficial da inflação brasileira.

Para $\pi_{t+4 \mid t}$, isto é, a medida de expectativa de inflação, utilizamos os dados diários disponibilizados pelo Banco Central acerca da mediana das expectativas de mercado para a inflação acumulada nos próximos doze meses. A partir desses dados diários, calculamos a média trimestral, que foi empregada em nossas estimativas. No entanto, tal série sobre as expectativas de mercado encontra-se disponível apenas a partir de dezembro de 2001, o que poderia constituir fator de limitação para o período de estimação de nossos modelos. Para contornarmos tal problema, para o período entre o primeiro trimestre de 2000 e quarto trimestre de 2001, utilizamos as expectativas centrais (i.e, do cenário básico) do Banco Central, descritas 
nos Relatórios de Inflação Trimestrais divulgados pela instituição ao longo dos anos 2000 e 2001.

Taxa de juros real, $r_{t}$, foi calculada de forma usual a partir da taxa de juros nominal e da taxa de inflação. Evidentemente, tivemos aqui um problema de não estacionariedade da série, dada a trajetória declinante da taxa de juros real em nosso passado recente. Para contornarmos tal problema, a variável incluída na estimação da curva IS foi a primeira diferença da taxa real. Como em nossa IS incluímos como variável explicativa o hiato do produto defasado com coeficiente igual a um, tal formulação implica que estamos explicando variações do hiato do produto a partir de variações do patamar da taxa de juros real.

Taxa de crescimento mundial: $w_{t}$. Sua inclusão na curva IS tem como objetivo captar os impactos sobre a produção doméstica de flutuações na demanda por exportações brasileiras. Assim, a proxy foi construída pela composição da taxa de crescimentos dos principais parceiros comerciais do Brasil, ponderados por sua importância relativa normalizada, trimestre a trimestre, na pauta de exportações brasileiras (em termos de valor). Mais especificamente, utilizamos as taxas de crescimento para Argentina, China, Estados Unidos e União Europeia. A utilização da participação relativa trimestre a trimestre, ao invés da média do período, foi relevante para dar conta da crescente importância da China como destino das exportações brasileiras. Para o período estudado (2002Q3 - 2013Q2), tais países foram em média responsáveis, conjuntamente, por $60,31 \%$ do total do valor exportado pelo Brasil. Somando a tal patamar expressivo o papel de termômetro da economia global de China, Estados Unidos e União Europeia, acreditamos que a proxy cumpre bem seu papel de captar pressões externas pelo canal da IS.

Preços externos: $e_{t} \mathrm{C}_{t}$. Como proxy para o vetor de transmissão de movimentos dos preços externos sobre a inflação doméstica, no contexto da curva de Phillips, empregamos a série de taxas de variação dos preços, do índice CRB excluindo-se o petróleo. Tal proxy foi utilizada para seguir a construção empregada pelo Banco Central do Brasil em sua curva de Phillips. ${ }^{11}$ A exclusão do petróleo é, ade-

${ }^{11}$ Relatório Trimestral de Inflação, Setembro de 2012. Box Revisão dos Modelos de Projeção de Pequeno Porte - 2012. Na página 97 de tal relatório, o Banco Central afirma que "a inflação externa passou a ser medida pelo índice Commodity Research Bureau (CRB), em substituição ao Índice de Preços ao Produtor dos Estados Unidos (PPI all commodities)." 
mais, plenamente justificada pelo controle dos preços domésticos do produto praticado pela Petrobrás, que possui monopólio virtual da distribuição dos derivados desse produto no Brasil. Assim, oscilações nos preços de petróleo no mercado internacional, embora tenham forte impacto sobre o CRB geral, têm impacto bastante reduzido, no curto prazo, sobre os preços domésticos.

\section{Anexo 2 - Derivação da Equação de Euler}

O problema do Banco Central é:

$\min _{i_{t}} \mathcal{L}=\left(\pi_{t+4}-\bar{\pi}_{t+4}\right)^{2}+\lambda_{y} y_{t+4}^{2}+\lambda_{i}\left(i_{t}-i_{t-1}\right)^{2}+\lambda_{e}\left(e_{t}-e_{t-1}\right)^{2}$

Sujeito à

$$
\begin{aligned}
& y_{t+k}=y_{t+k-1}+\beta_{1}\left(r_{t+k-2}-r_{t+k-3}\right)+ \\
& \beta_{2}\left(q_{t+k-2}\right)+\beta_{3}\left(w_{t+k}-w_{t+k-1}\right)+\epsilon_{t+k}^{y} \\
& \pi_{t+k}=\pi_{t+k-1}+\alpha_{1}\left(y_{t+k-2}\right)+\alpha_{2}\left(e_{t+k-2} C_{t+k-2}\right)+\epsilon_{t+k}^{\pi} \\
& q_{t+k+1}=q_{t+k}+\epsilon_{t+k+1}^{q}
\end{aligned}
$$

Para facilitar a exposição, derivaremos separadamente os termos aditivos da equação de perda $\mathcal{L}$. Na sequência, somaremos todos os termos obtidos para explicitar a equação de Euler a ser estimada por GMM. Vale observar que, embora estejamos seguindo a metodologia aplicada por D'Adamo (2010), a equação de Euler aqui derivada se afasta daquela obtida pelo autor em função de diferenças nos modelos empíricos obtidos. Além disso, o autor não considera o impacto da taxa de juros sobre a taxa de câmbio nominal e, portanto, sobre o preço em moeda local do índice de commodities. Consideramos esse fator relevante para o caso brasileiro e, portanto, o incluímos na derivação apresentada na sequência.

Já na nota de rodapé de número 5 , presente na página 88 , é explicitado que o "índice do CRB utilizado - CRB BLS Spot Index - agrega as cotações no mercado à vista de 22 commodities agrícolas e metálicas (não inclui petróleo)". 
Assim, minimizando a função de perda sob o conjunto de restrições descrito, temos que:

(i) $\min _{\mathrm{i}_{\mathrm{t}}} \delta^{4}\left[\left(\pi_{\mathrm{t}+4 \mid \mathrm{t}}-\bar{\pi}_{t+4}\right)^{2}\right]$

$\Rightarrow 2 \delta^{4}\left(\pi_{\mathrm{t}+4 \mid \mathrm{t}}-\bar{\pi}_{t+4}\right)\left[\frac{\partial \pi_{\mathrm{t}+4}}{\partial \mathrm{y}_{\mathrm{t}+2}} \frac{\partial \mathrm{y}_{\mathrm{t}+2}}{\partial \mathrm{i}_{\mathrm{t}}}+\frac{\partial \pi_{\mathrm{t}+4}}{\partial \mathrm{y}_{\mathrm{t}+2}} \frac{\partial \mathrm{y}_{\mathrm{t}+2}}{\partial \mathrm{q}_{\mathrm{t}}} \frac{\partial \mathrm{q}_{\mathrm{t}}}{\partial \mathrm{i}_{\mathrm{t}}}+\frac{\partial \pi_{\mathrm{t}+4}}{\partial \pi_{\mathrm{t}+3}} \frac{\partial \pi_{\mathrm{t}+3}}{\partial \pi_{\mathrm{t}+2}} \frac{\partial \pi_{\mathrm{t}+2}}{\partial\left(\mathrm{e}_{\mathrm{t}} C_{t}\right)} \frac{\partial\left(\mathrm{e}_{\mathrm{t}} C_{t}\right)}{\partial \mathrm{i}_{\mathrm{t}}}\right]=0$

$\Rightarrow 2 \delta^{4}\left(\pi_{\mathrm{t}+4 \mid \mathrm{t}}-\bar{\pi}_{t+4}\right)\left(\alpha_{1} \beta_{1}+\alpha_{1} \beta_{2}+\alpha_{2}(-1)\right)=0$

Definindo $\mathrm{k}_{1} \equiv \alpha_{1}\left(\beta_{1}+\beta_{2}\right)-\alpha_{2}$,

$$
2 \delta^{4}\left(\pi_{\mathrm{t}+4 \mid \mathrm{t}}-\bar{\pi}_{t+4}\right) \mathrm{k}_{1}=0
$$

(ii) $\min _{i_{\mathrm{t}}} \delta^{4} \lambda_{\mathrm{y}} y_{\mathrm{t}+4 \mid \mathrm{t}}^{2}$

$\Rightarrow 2 \delta^{4} y_{t+4} \lambda_{y}\left(\frac{\partial \mathrm{y}_{\mathrm{t}+4}}{\partial \mathrm{y}_{\mathrm{t}+3}} \frac{\partial \mathrm{y}_{\mathrm{t}+3}}{\partial \mathrm{y}_{\mathrm{t}+2}} \frac{\partial \mathrm{y}_{\mathrm{t}+2}}{\partial \mathrm{i}_{\mathrm{t}}}+\frac{\partial \mathrm{y}_{\mathrm{t}+4}}{\partial \mathrm{y}_{\mathrm{t}+3}} \frac{\partial \mathrm{y}_{\mathrm{t}+3}}{\partial \mathrm{y}_{\mathrm{t}+2}} \frac{\partial \mathrm{y}_{\mathrm{t}+2}}{\partial \mathrm{q}_{\mathrm{t}}} \frac{\partial \mathrm{q}_{\mathrm{t}}}{\partial \mathrm{i}_{\mathrm{t}}}\right)=0$

$\Rightarrow 2 \delta^{4} y_{t+4 \mid t} \lambda_{y}\left(\beta_{1}+\beta_{2}\right)=0$

Definindo $k_{2} \equiv \beta_{1}+\beta_{2}$

$$
2 \delta^{4} y_{t+4 \mid t} \lambda_{y} \mathrm{k}_{2}=0
$$

(iii) $\quad \min _{\mathrm{i}_{\mathrm{t}}} \lambda_{\mathrm{i}}\left(\mathrm{i}_{\mathrm{t}}-\mathrm{i}_{\mathrm{t}-1}\right)^{2}$

$$
\Rightarrow 2 \lambda_{i}\left(i_{t}-i_{t-1}\right)=0
$$

(iv) $\min _{\mathrm{i}_{\mathrm{t}}} \lambda_{e}\left(e_{\mathrm{t}}-e_{\mathrm{t}-1}\right)^{2}$

$$
\Rightarrow-2 \lambda_{e}\left(e_{t}-e_{t-1}\right)=0
$$


Somando (i) - (iv), temos que:

$\delta^{4}\left(\pi_{\mathrm{t}+4 \mid \mathrm{t}}-\pi\right) k_{1}+\delta^{4} y_{t+4 \mid t} \lambda_{y} k_{2}+\lambda_{i}\left(i_{t}-i_{t-1}\right)-\lambda_{e}\left(e_{t}-e_{t-1}\right)=0$

Logo, obtemos

$i_{t}-i_{t-1}+\left(\delta^{4} \mathrm{k}_{1}\right) \frac{1}{\lambda_{i}}\left(\pi_{\mathrm{t}+4 \mid \mathrm{t}}-\pi\right)+\left(\delta^{4} \mathrm{k}_{2}\right) \frac{\lambda_{y}}{\lambda_{i}} y_{t+4 \mid t}-\frac{\lambda_{e}}{\lambda_{i}}\left(e_{t}-e_{t-1}\right)=0$

que é a condição de momento sobre a qual aplicamos o GMM. Mais precisamente, o modelo estimado pode ser escrito como

$i_{t}-i_{t-1}+0,0209 \phi_{1}\left(\pi_{\mathrm{t}+4 \mid \mathrm{t}}-\pi\right)-0.1677 \phi_{2} y_{t+4 \mid t} \phi_{2}-\phi_{3}\left(e_{t}-e_{t-1}\right)=0$

Onde $\phi_{1}=\frac{1}{\lambda_{i}}, \phi_{2}=\frac{\lambda_{y}}{\lambda_{i}}$ e $\phi_{3}=\frac{\lambda_{e}}{\lambda_{i}}$. Vale observar que utilizando tal formulação não encontramos nenhum problema para identificação dos parâmetros de preferência do Banco Central. 